\title{
Time-varying interference spectral analysis for Cognitive UWB networks
}

\author{
Massimo Francone, Daniele Domenicali, Maria-Gabriella Di Benedetto \\ School of Engineering, University of Rome La Sapienza \\ Infocom Department, Via Eudossiana, 18 - 00184 Rome, Italy \\ email: \{massimo,dome,dibenedetto\}@newyork.ing.uniroma1.it
}

\begin{abstract}
The potential interference from and to nearby wireless networks makes coexistence a crucial task in the design of Ultra Wide Band (UWB) systems. UWB radio technology may surely benefit from the use of Cognitive Radio (CR) techniques, implementing collaborative coexistence schemes. In this work, we consider a network formed by self-organizing nodes that operate according to the UWB principle. A Conscious Node (CNode) acts as the coordinator of the network and implements cognitive rules. Spectrum sensing is necessary for the CNode to be aware of the RF context and of network state. We provide an analysis method for the internal UWB interference experienced at the CNode receiver, by making use of the short-term Fourier transform based on windowing. We show that by adopting particular windows, such as the Blackman window, specific characteristics of the sensed interference spectrum may be highlighted. This work provides a first step towards reliable models for network spectral evolution that may provide the basis for advanced predictive and cognitive mechanisms.
\end{abstract}

\section{INTRODUCTION}

The most salient feature of Ultra Wide Band (UWB) radio signals is their ultra wide bandwidth. As such, these signals occupy ranges of frequencies that are in principle already in use for other purposes and services. Possible interference caused by UWB signals may, therefore, generate serious concern. UWB systems, in turn, may suffer from interference from other radio signals. As a consequence, UWB emitted radiations are limited to- or are most likely to be limited to- regulated values that strongly contain the emitted power of radiations.

UWB regulation by the Federal Communications Commission (FCC, 2002) in the United States [1] has opened the way to the concept in large of context-aware radio based on coexistence. This principle fully fits with the emerging innovative concept of "cognitive radio" aimed at defining and developing technologies that can enable a radio device to adapt its spectrum according to the operating environment, that is, to be aware of the scenario in which it operates. According to [2], in which the cognitive principle was first introduced, a cognitive radio behaves according to five main actions: observe, plan, decide, learn, and act, in order to form a cognition cycle, as illustrated by Fig. 1. The final goal remains to form wireless networks that cooperatively coexist with other wireless networks and devices.

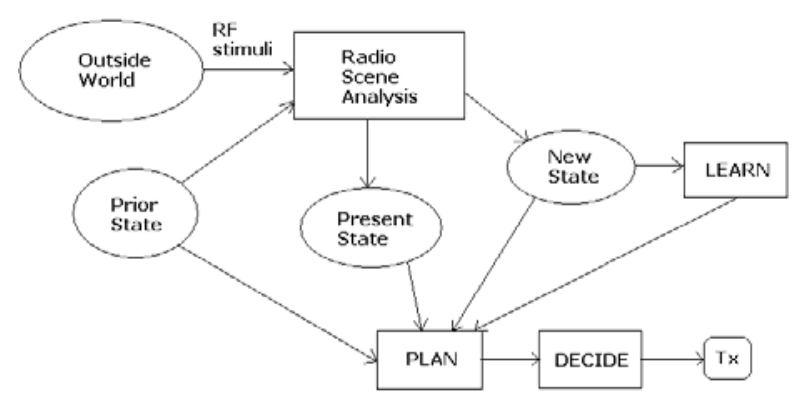

Figure 1 - Cognition cycle for a cognitive wireless device. The device operates according to 5 main actions: observe, plan, decide and act.

Within this framework, an ambitious goal is the design and development of smart UWB devices able to adapt themselves to the environment, whether this refers to channel or interference patterns, by changing spectral shape and features of radiated signals while maintaining compatibility with regulations on emitted radiations.

Introducing cognitive principles in an UWB self-organizing network requires that at least one of the UWB nodes is able to implement the cognitive paradigm, and of acting as the coordinator. This node, that we call the Conscious Node (CNode), as first introduced in [3], must be context aware; It evaluates and selects one strategy of operation on the basis of the Radio Frequency (RF) stimuli that it perceives. By performing spectrum sensing over a wide bandwidth, the CNode must be able to match its internal models to external observations, and to adapt its strategy of operation as a function of both internal and external unpredictable events. Modelling spectral characteristics of interference is thus a crucial problem in the design.

The purpose of this work is to provide an analysis method for modelling spectral interference that occurs at the receiver of a cognitive device belonging to an UWB network.

We investigate spectral characteristics of interference 
patterns formed by UWB pulses, by making use of the shortterm Fourier transform based on windowing.

Network evolution is examined from a statistical point of view: births and deaths of active nodes are modelled as a Markov chain characterized by pre-established transition probability matrices.

In a condition of rapid changes in network evolution, the CNode should operate at a high sampling rate. When simulating spectrum sensing, we may want therefore to approximate continuous phenomena, and as a consequence set observation times to very short windows.

The paper is organized as follows. Section II illustrates the reference system model. Section III focuses on spectrum estimation and analyzes the effects of windowing the signal. Section IV discusses interference sensing and provides information about signal format. Simulation results and a comparison of performance obtained using different windows are presented in Section V. Section VI addresses the conclusions, and drafts future research topics.

\section{REFERENCE SYSTEM MODEL}

We make reference to a network formed by self-organizing nodes that operate according to the UWB principle. A Conscious Node (CNode) acts as the coordinator of the network and implements cognitive rules.

The RF stimuli perceived by the CNode are non-stationary spatial and temporal signals. Spectrum sensing must be thus performed by time-frequency analysis of the RF environment. The incoming RF stimuli are segmented into a continuous sequence of frames forming interference patterns. Each segment is considered to be a pseudo-stationary signal, and is analysed in the frequency domain.

Modelling a node in a wireless cognitive network implies the definition of both discrete and continuous dynamics. A common adopted approach assumes that all cognitive operations occur at clock beats or multiples. Changes in environment, however, may be continuous-like, forcing the CNode to operate at a high sampling rate. A lack of synchronism between network states evolution and environmental changes (e.g., atmospheric changes) must be taken into account in the definition of a cognitive mechanism for network control.

A mathematical model that appropriately defines discrete and continuous systems is provided by hybrid system theory. Hybrid models describe systems composed of both continuous and discrete variables. The interactions of these variables determine the hybrid system evolution. Therefore, from a hybrid system point of view, we can see the network state as a discrete-state automaton perturbed by continuous variables. Mechanisms that govern and determine transitions between discrete states are very complex and have been recently the subject of intense research [3].

Spectrum sensing at the CNode must be performed on a short time interval basis in order to track the continuous variations of the RF environment. In addition, the observation time must be sufficiently long to produce accurate spectral estimates.

In the above scenario, internal interference is caused by the presence of multiple active users in the same network of the CNode, that is by Multi-User Interference (MUI). Internal interference at the CNode consists thus in a set of TH-PPMUWB pulses that are randomly distributed, and create a particular interference pattern within a single observation interval (temporal burst). Based on interference spectral analysis, the CNode may adapt its behaviour to the current network topology and status. Most important concept is the time scale over which this adaptation takes place.

On the other hand, external interference is caused by the presence of surrounding traditional Wireless networks such as Wireless Local Area Networks 802.11-like (WLAN or WiFi), 802.15 (Bluetooth), or $2 \mathrm{G} / 3 \mathrm{G}$ cellular networks.

\section{SHORT TIME ANALYSIS}

In the spectral estimation literature, it is well known that spectrum estimation problem is made difficult by the biasvariance dilemma $[4,5]$.

A classical spectrum estimator for stationary signals, the periodogram, is defined as simply the squared magnitude of the Fourier transform of a windowed version of the data signal. The window function reduces the side lobes leakage phenomenon but increases the variance of the estimate. This variance improvement is due to the loss of information resulting from a reduction in the effective sample size.

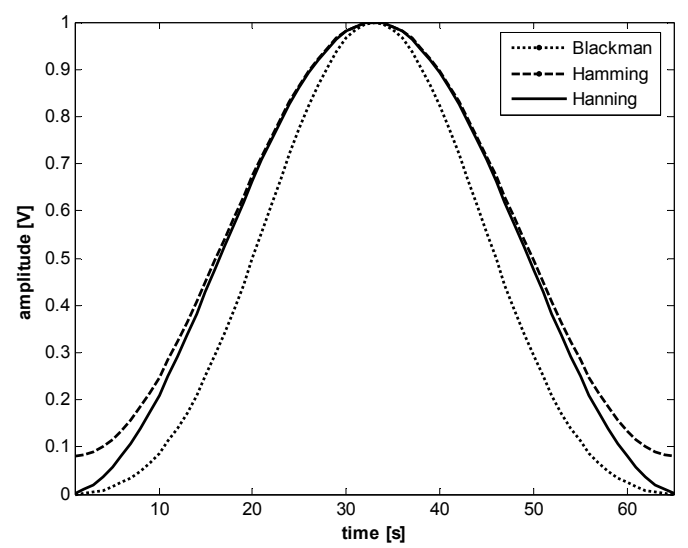

Figure 2 - Most commonly used windows: Hamming window (dashed line), Hanning window (continuous line), and Blackman window (dotted line).

While the periodogram suffers from large variance, this variance can be reduced by cutting the signal samples into blocks, computing a periodogram of each block, and then averaging the periodograms obtained (Welch method [6]). The signal segments can be windowed before calculating the periodogram to increase the quality of power spectrum estimates. This procedure, however, also smears and biases the resulting spectrum estimate. The bias-variance trade-off is clear: reducing the variance necessitates averaging over a larger number of shorter blocks, which increases the bias. The side lobes leakage phenomenon is mitigated by tapering 
(i.e., windowing) the time signal samples smoothly to zero at each end. The most common adopted windows are the Hamming, Hanning, and Blackman windows, as shown in Fig. 2. The Kaiser window is defined as follows:

$$
w[n]= \begin{cases}\frac{I_{0}\left[\beta\left(1-[(n-\alpha) / \alpha]^{2}\right)^{1 / 2}\right]}{I_{0}(\beta)}, & 0 \leq n \leq M \\ 0, & \text { otherwise }\end{cases}
$$

where $\alpha=M / 2$ and $I o(\cdot)$ represents the zero ${ }^{\text {th }}$-order modified Bessel function of the first kind. In contrast to the other windows, the Kaiser window has two parameters: the length $(M+1)$ and the shape parameter $\beta$ (see Fig.3). By varying $(M+1)$ and $\beta$, the window length and the shape can be adjusted to trade spectral characteristic of the window.

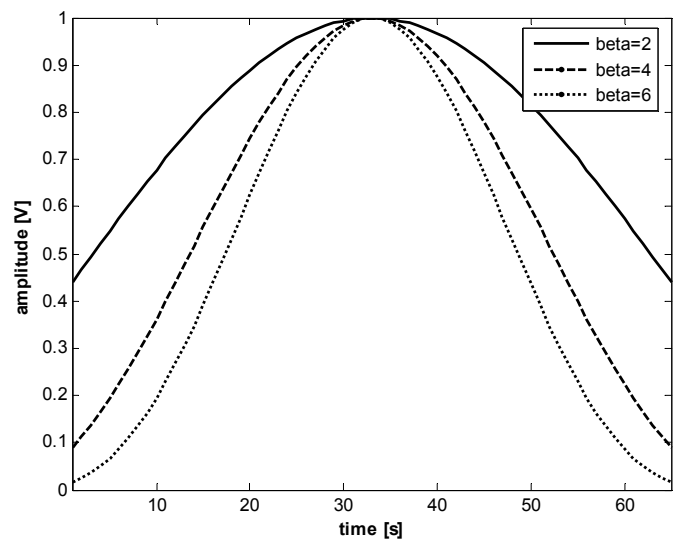

Figure 3 - Kaiser window with different $\beta: \beta=2$ (continuous line), $\beta=4$ (dashed line), $\beta=6$ (dotted line)

\section{INTERFERENCE SENSING}

To obtain information about internal interference, the CNode must perform a spectral analysis after tapering the whole signal by means of a given window. We will consider the Hamming, Hanning, Blackman, and Kaiser windows.

The UWB signal format is the one typical of Impulse Radio (IR) signals, with Time-Hopping coding (TH) and binary Pulse Position Modulation (PPM), and can be described by the following expression [6]:

$$
s(t)=\sqrt{P_{T X} T_{S}} \sum_{j} p_{w}\left(t-j T_{s}-c_{j}-a_{j} \varepsilon\right)
$$

where $P_{T X}$ is the average transmitted power, $T_{S}$ is the pulse repetition period, $p_{w}(t)$ is the energy normalized pulse shape, $c_{j}<T_{S}$ is the TH value for pulse $j, a_{\mathrm{j}}$ is the data symbol carried by pulse $j$, and $\varepsilon$ is the PPM shift.

We can consider in general each interference pattern as the realization of a stochastic process: the arrival times of pulses is modelled as a Poisson arrival process with rate $\lambda$. Therefore, the number of pulses in a finite time interval obeys the Poisson $(\lambda t)$ distribution:

$$
\operatorname{prob}\{N(t)=n\}=\frac{(\lambda t)^{n}}{n !} e^{-\lambda t}
$$

The considered pulse waveform is the second derivative of a Gaussian pulse [6]. Pulse amplitudes are assumed to be statistically independent uniform random variables over $[0$, 0.015] Volts. In this manner, we take into account reasonable multi-path effects in the propagation over the channel.

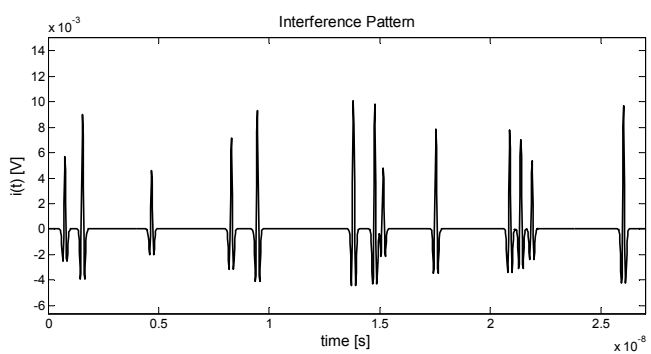

Figure 4 - Example of interference pattern made of UWB pulses. Pulse waveforms are second derivative Gaussian. Arrival times are generated according to a Poisson stochastic process.

\section{EXPERIMENTAL SET-UP}

\section{a. Results of simulations}

At the CNode receiver, within each observation time interval, we find a set of TH-PPM-UWB pulses that are randomly distributed (they belong to multiple users with different Time Hopping codes, forming a particular interference pattern, as shown in Fig.4).

Network evolution is examined from a statistical point of view: births and deaths of active nodes are modelled as a Markov chain characterized by pre-established transition probabilities matrices.

In a condition of rapid changes in network evolution, the CNode should operate at high sampling rate. Therefore, we want to simulate a spectrum sensing as continuous as possible, setting the observation time to $27 \mathrm{~ns}$.

The first aspect under investigation is the analysis of variations in spectrum estimation caused by the use of different applied windows. The first set of simulations has served to approach the problem from a statistical point of view. The two basic steps of the simulation procedure are the following:

1) Generation of an interference UWB pattern according to:

- a Poisson stochastic process for the pulse arrival times, governed by a specific Poisson parameter $\lambda$;

- $\quad$ statistically independent uniform random variables over [0, 0.015] Volts for pulse amplitudes.

2) Evaluation of the Power Spectral Density (PSD) applying the Welch method [7] using three 50\% overlapping segments. In particular, each segment has been chosen as follows:

- it contains half number of samples compared to the original signal;

- $\quad$ has been windowed with a specific window. 
Note that as $\lambda$ (average pulse inter-arrival frequency) increases, we have a higher number of interfering pulses, that is, a higher number of nodes are active in the network.

The adopted characteristics for these interference patterns are the following:

- $\quad$ the adopted pulse waveform is the second derivative Gaussian pulse;

- $\quad T_{m}=0.5 n s$ (time length of each interfering pulse);

- $\quad \alpha=0.25 n s$

- $\quad T_{o s s}=27 n s$ (time length of interference pattern).

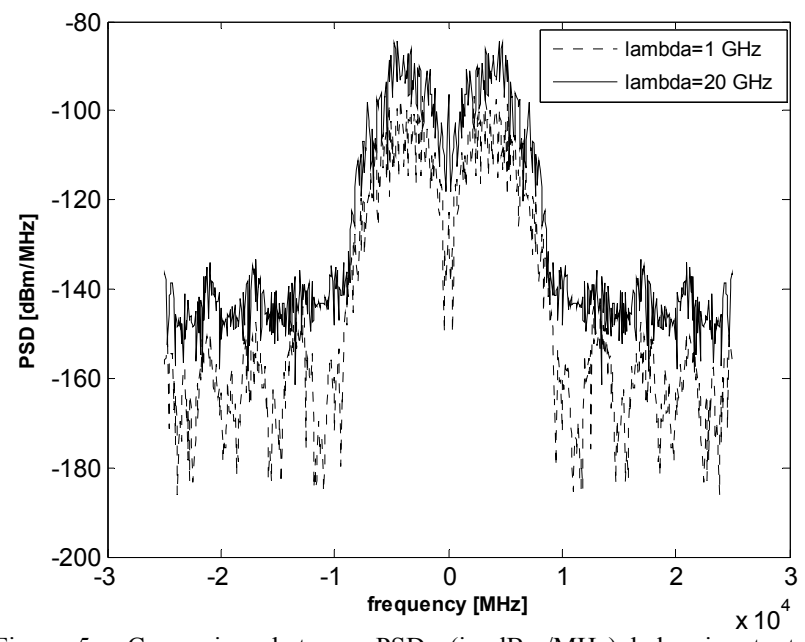

Figure 5 - Comparison between PSDs (in $\mathrm{dBm} / \mathrm{MHz}$ ) belonging to two different interference patterns characterized by $\lambda=1 \mathrm{GHz}$ (continuous line) vs. $\lambda=10 \mathrm{GHz}$ (dotted lines), computed using the Hamming window

Several configurations of interference UWB patterns governed by specific $\lambda$ values were analysed using the windows shown in Figs.2 and 3, in order to find possible interference prediction criteria.

One result was that the CNode could predict the number of interfering pulses, that is, the state of interference in a given time instant, by processing the 'level' of the sensed spectrum. It was found in fact that when the Poisson parameter $\lambda$ increases (i.e., the average number of interfering pulses increases) the PSD moves upward. Figure 5, where the PSDs of two different interference patterns are shown: the Poisson parameter $\lambda$ is set to $1 \mathrm{GHz}$ and $20 \mathrm{GHz}$, and the window applied to compute the PSD is the Hamming window.

A more detailed study was performed by generating 2000 interference UWB patterns all characterized by a single $\lambda$ value. The PSD of each interference pattern was computed by means of the Welch method described above. The signal segments were windowed using different windows. The following statistical quantities were observed:

1) Average PSD maximum value;

2) Average PSD minimum value;

3) Average PSD min-max.
TABLE I - Average PSD maximum, minimum, and minmax values for different window types

\begin{tabular}{llll}
\multicolumn{1}{c}{$\lambda$} & $\begin{array}{l}\text { Average } \\
\text { PSD } \\
\text { maximum }\end{array}$ & $\begin{array}{l}\text { Average PSD } \\
\text { minimum }\end{array}$ & $\begin{array}{l}\text { Average } \\
\text { max-min gap }\end{array}$ \\
\hline $0.1 \mathrm{GHz}$ & $-166.37 \mathrm{dBm}$ & $-256.23 \mathrm{dBm}$ & $90.22 \mathrm{dBm}$ \\
\hline $0.5 \mathrm{GHz}$ & $-154.60 \mathrm{dBm}$ & $-251.50 \mathrm{dBm}$ & $96.90 \mathrm{dBm}$ \\
\hline $1 \mathrm{GHz}$ & $-151.09 \mathrm{dBm}$ & $-248.68 \mathrm{dBm}$ & $97.58 \mathrm{dBm}$ \\
\hline $10 \mathrm{GHz}$ & $-141.69 \mathrm{dBm}$ & $-239.54 \mathrm{dBm}$ & $97.85 \mathrm{dBm}$ \\
\hline $50 \mathrm{GHz}$ & $-138.58 \mathrm{dBm}$ & $-236.56 \mathrm{dBm}$ & $97.84 \mathrm{dBm}$
\end{tabular}

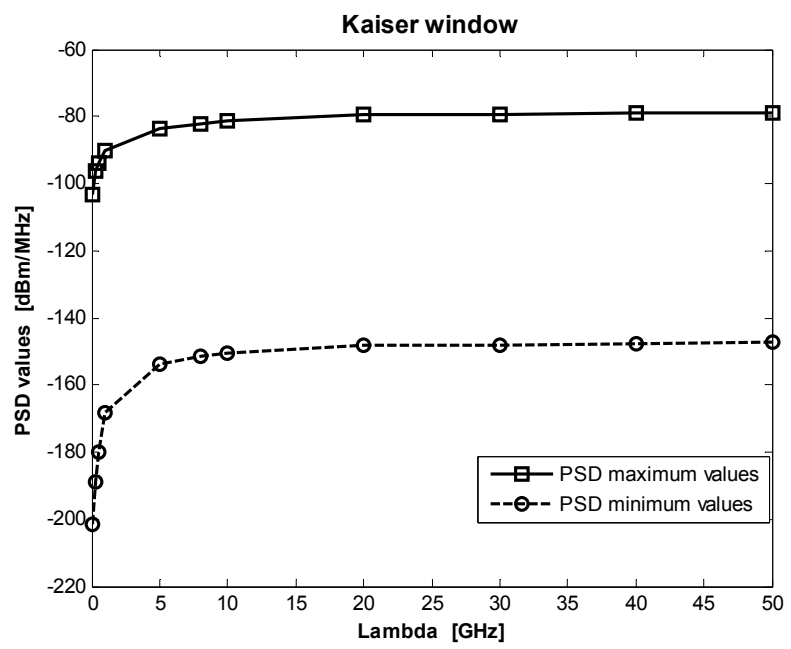

Figure 6 - Average PSD maximum (continuous line) and minimum (dashed line) values vs. Poisson parameter $\lambda$. PSDs have been computed using Welch's method and the Kaiser window $(\beta=1)$.

As reported in Table I values for the above quantities confirm the 'translation effect' as shown.

\section{B. The Kaiser case}

It is worth to highlight the results obtained by application of the Kaiser window. Kaiser windows with different $\beta$ were tested. Detailed analysis of the results was carried out for different $\beta$ values, and are reported here in the case $\beta=1$.

Figure 6 shows that the PSD average peak and minimum values, where the $\mathrm{x}$-axis represents $\lambda$ in $G H z$, i.e. average number of interfering pulses occurred in each ns, while the $y$ axis represents the investigated PSD parameter in $\mathrm{dBm}$.

Results indicate that PSD maximum and minimum values increase with $\lambda$ up to a value for $\lambda$ of about $10 \mathrm{GHz}$, to reach then a plateau.

Figure 7 shows the average PSD min-max gap as a function of $\lambda$ : the shape of this function resembles the exponential function $e^{-x}$. It is important to note that this property was only observed in the case of a Kaiser window with $\beta=1$, and makes the Kaiser window an interesting window for interference modelling purposes. 


\section{Energy considerations}

A final result regards energy considerations in windowing on the analysed UWB signals. The Energy Spectral Density (ESD) was computed using the Welch method and four different windows. The energy located at frequencies above $10 \mathrm{GHz}$ was considered as out-of-band energy.

The simulation procedure can be summarized in 3 steps:

- generation of an interference pattern, single occurrence of a Poisson stochastic process;

- computation of seven ESD's by applying the Welch method with seven different windows;

- analysis of results.

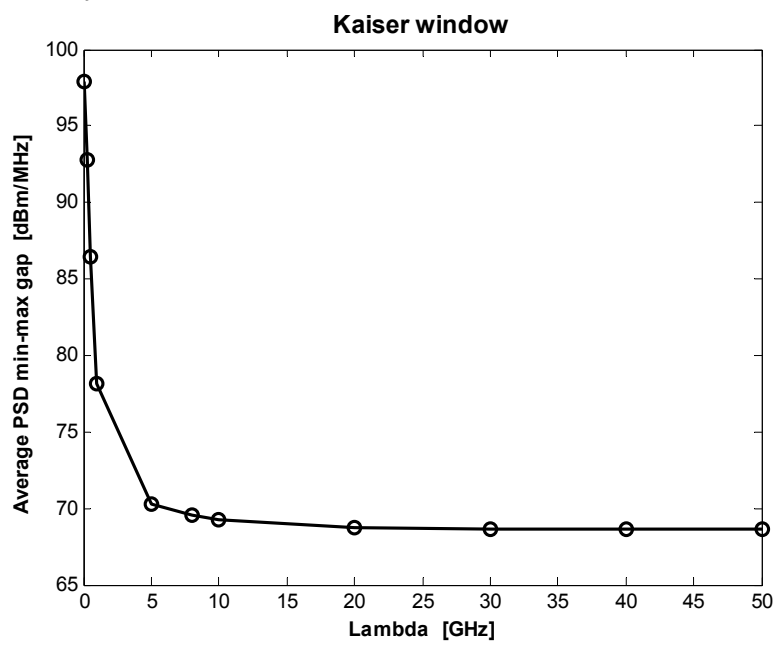

Figure 7 - Average PSD min-max gap vs. Poisson parameter $\lambda$. PSDs were computed by means of the Welch method, using a Kaiser window $(\beta=1)$.

In order to obtain results with a sufficient statistical accuracy, the procedure included 5000 Poisson random occurrences (i.e. interference patterns).

In the third step "analysis of results" those windows that verify particular condition can be found. In particular, we analyzed the number of occurrences by which with a particular window one obtained the smallest out-of-band energy. Results are presented in Table II. According to these data, the Blackman window is statistically characterized by the lowest energy contribution at frequencies above $10 \mathrm{GHz}$ (maximum value of $\min$ Eout).

This property makes the Blackman window attractive for interference modelling purposes.

TABLE II - Percentage of occurrences with minimum outof-band energy

\begin{tabular}{cc} 
Window type & \% min_Eout \\
\hline Hamming & 5.26 \\
\hline Blackman & 43.98 \\
\hline Kaiser with $\beta=1$ & 25.66 \\
\hline Hanning & 25.10
\end{tabular}

\section{CONCLUSIONS}

Spectrum sensing is crucial in order for a cognitive node to be aware of the RF context and of network state.

In this paper, we showed that by adopting particular windows, such as the Kaiser window, specific characteristics of a sensed spectrum of interference is obtained. This study is important in order to model internal interference occurring in a UWB network of nodes.

In particular, we generated interference UWB patterns according to Poisson stochastic processes for the pulse arrival times (governed by a specific Poisson parameter $\lambda$ ) and statistically independent uniform random variables over $[0,0.015]$ Volts for pulse amplitudes. We evaluated the PSD by applying the Welch method [7] averaging over three 50\% overlapping segments. In particular, each segment was such as it would contain half the number of samples compared to the original signal, and the signal in each segment was windowed by means of one of the following window: Hamming, Hanning, Kaiser, Blackman, and Kaiser.

Results were presented in terms of maximum, minimum, and min-max values for the PSD of interference. Results indicate that the window choice may have significant impact on the estimation of the interference PSD pattern in the case of UWB impulse radio interfering nodes, and suggest the adoption of the Kaiser and the Blackman windows for UWB spectrum estimation. In particular, whereas the Kaiser window manifests attractive properties as the number of interfering UWB pulses increases, the Blackman window shows the lowest energy contribution of the spectrum sensed at very high frequencies.

In conclusion, we want to underline the concept that the specific application of interest can adopt the window that better matches with its UWB interference modeling purposes.

The final goal of this work is the identification of network states that may be associated to particular spectral features of the sensed spectrum. By this way, a CNode might predict the spectrum of internal interference by knowing the present network state. Based on this prediction, the CNode may then proceed in estimating external interference as the difference between the real sensed and predicted spectra.

\section{ACKNOWLEDGEMENTS}

This work was partially supported by the European Union under the 6th Framework Network of Excellence HYCON (contract number FP6-IST-511368).

\section{REFERENCES}

[1] Federal Communications Commission, 2002, "Revision of Part 15 of the Commission's rules Regarding UltraWideband Transmission Systems: First report and order," 
Technical Report FCC 02-48 (adopted February, 14 2002; released April 22, 2002).

[2] Mitola J. and Maguire G.Q., "Cognitive radio: Making software radios more personal," IEEE Pers. Commun., vol. 6, no. 4, pp. 13-18, Aug. 1999.

[3] Di Benedetto M.-G., Giancola G. and Di Benedetto M.D, 2006, "Introducing Consciousness in UWB networks by Hybrid Modelling of Admission Control," to appear in Special Issue on "Ultra Wide Band for Sensor Networks" of the ACM/Springer Journal on Mobile Networks and Applications, (2006).

[4] Bayram M. and Baraniuk R.G., "Multiple window timevarying spectrum estimation," in Nonlinear and Nonstationary signal processing, pp. 292-316. Cambridge University press, 2000.

[5]. Thomson, D. J., "Spectrum estimation and harmonic analysis," Proceedings of the IEEE, vol. 20, pp. 1055-1096, Sep. 1982.

[6]. Di Benedetto, M.-G. and Giancola G., Understanding Ultra Wide Band Radio fundamentals, Prentice Hall, 2004.

[7] Welch P. D., "The Use of Fast Fourier Transform for the Estimation of Power Spectra: A Method Based on Time Averaging Over Short, Modified Periodograms," IEEE Transactions on Audio and Electroacoustics, 1967, 15, 70-3. 